\title{
FORMAÇÃO INICIAL DE PROFESSORES PARA USO DAS TECNOLOGIAS: A APROPRIAÇÃO DO CONHECIMENTO TECNOLÓGICO EXPRESSO NO PROJETO PEDAGÓGICO DE CURSO
}

\author{
Juliane Colling ${ }^{1}$ \\ Marciane Maciel $^{2}$ \\ Luiz Martins Junior ${ }^{3}$ \\ Sibele Mueller ${ }^{4}$ \\ Luana Angélica Alberti ${ }^{5}$
}

\section{Resumo}

Interagimos diariamente com diversos recursos tecnológicos em diversos ambientes e situações, e o meio escolar não foge à esta regra. Para tanto, a fim de utilizar estes recursos de forma a modificar as práticas educativas, é de grande importância que os professores tenham conhecimento crítico e reflexivo para articulação dos recursos tecnológicos às finalidades pedagógicas de ensino de conteúdos específicos. Nessa perspectiva, este trabalho tem por objetivo evidenciar de que forma as tecnologias se articulam às atividades pedagógicas desenvolvidas em um curso de licenciatura, tomando por base de análise do Projeto Pedagógico de Curso de Pedagogia da Universidade Federal da Fronteira Sul. A partir da análise realizada percebe-se o PPC analisado já prevê a articulação de conhecimentos espcíficos, pedagógicos e tecnológicosas,

destacando algu

\footnotetext{
1 Mestranda em Educação - Universidade Federal da Fronteira Sul. julianecolling_gti@hotmail.com.

2 Mestranda em Educação - Universidade Federal da Fronteira Sul. marciani_maciel@hotmail.com.

3 Doutorando em Geografia - Universidade Federal de Santa Catarina. luizmartins.jr@hotmail.com.

${ }^{4}$ Pós Graduanda em Gestão de Banco de Dados - Universidade Luterana do Brasil. sibelemueller@hotmail.com.

5 Mestranda em Educação - Universidade Federal da Fronteira Sul. luana.albertti@yahoo.com.br.
}

Criar Educação, Criciúma, v. 6, ํo2, julho/novembro 2017.- PPGE - UNESC 


\title{
FORMAÇÃO INICIAL DE PROFESSORES PARA USO DAS TECNOLOGIAS: A APROPRIAÇÃO DO CONHECIMENTO TECNOLÓGICO EXPRESSO NO PROJETO PEDAGÓGICO DE CURSO
}

\author{
Juliane Colling ${ }^{1}$ \\ Marciane Maciel $^{2}$ \\ Luiz Martins Junior ${ }^{3}$ \\ Sibele Mueller ${ }^{4}$ \\ Luana Angélica Alberti ${ }^{5}$
}

\section{Resumo}

Interagimos diariamente com diversos recursos tecnológicos em diversos ambientes e situações, e o meio escolar não foge à esta regra. Para tanto, a fim de utilizar estes recursos de forma a modificar as práticas educativas, é de grande importância que os professores tenham conhecimento crítico e reflexivo para articulação dos recursos tecnológicos às finalidades pedagógicas de ensino de conteúdos específicos. Nessa perspectiva, este trabalho tem por objetivo evidenciar de que forma as tecnologias se articulam às atividades pedagógicas desenvolvidas em um curso de licenciatura, tomando por base de análise do Projeto Pedagógico de Curso de Pedagogia da Universidade Federal da Fronteira Sul. A partir da análise realizada percebe-se o PPC analisado já prevê a articulação de conhecimentos espcíficos, pedagógicos e tecnológicosas, destacando algumas possibilidades de articulação de

\footnotetext{
1 Mestranda em Educação - Universidade Federal da Fronteira Sul. julianecolling_gti@hotmail.com.

${ }_{2}$ Mestranda em Educação - Universidade Federal da Fronteira Sul. marciani_maciel@hotmail.com.

3 Doutorando em Geografia - Universidade Federal de Santa Catarina. luizmartins.jr@hotmail.com.

4 Pós Graduanda em Gestão de Banco de Dados - Universidade Luterana do Brasil. sibelemueller@hotmail.com.

5 Mestranda em Educação - Universidade Federal da Fronteira Sul. luana.albertti@yahoo.com.br.
}

Criar Educação, Criciúma, v. 6, ํo2, julho/novembro 2017.- PPGE - UNESC 
mas possibilidades de articulação de conhecimentos por meio de atividades interativas que estimulam a reflexão, debate e planejamento de práticas pedagógicas utilizando-se desses recursos.

\section{Abstract}

We interact daily with many technological resources in a lot of places and situations, and the school environment is not exception to this rule. Therefore, in order to use these resources to change the educational practices, it is of great importance that teachers have critical and reflexive knowledge to joint technological resources to the educational purposes of specific teaching content. In this perspective, this work aims to show how the technologies are linked to educational activities in an undergraduate course, based on analysis of the Pedagogical Course of Education of the Federal University of Southern Border Project. From the analysis performed PPC already analyzed it is clear provides the articulation of specific, pedagogical and technological knowledge, highlighting some possibilities of articulation of knowledge through interactive activities that stimulate reflection, debate and planning pedagogical practices using these resources.

Keywords: Initial Teacher Training, digital technologies of information and communication, TPACK.

\section{Introdução}

Interagimos diariamente com diversos recursos tecnológicos em diversos ambientes e situações e o meio escolar não foge a esta regra. A fim de utilizar estes recursos de forma a contribuir nas práticas educativas, é importante que os professores tenham conhecimento crítico e reflexivo para articulação dos recursos tecnológicos às finalidades pedagógicas de ensino de conteúdos. Neste sentido, este trabalho propõe-se a abordar algumas possibilidades de formação para uso pedagógico das tecnologias advindas das ações formativas promovidas em cursos de licenciatura, procedendo, para tal, uma análise do Projeto Político Pedagógico do curso de Pedagogia da Universidade Federal da Fronteira Sul, tomando como base teórica os conceitos de tecnologias da inteligência de Lévy (1993) e do Conhecimento Tecnológico, Pedagógico e de Conteúdo (TPACK) de Shulman (1986).

O caminho metodológico utilizado na realização desta pesquisa baseiase principalmente na pesquisa bibliográfica, buscando-se a construção de uma base teórica consistente, com contribuições de autores e pesquisadores importantes da área temática, e a análise de documento de domínio público, Criar Educação, Criciúma, v. 6, n²2, julho/novembro 2017.-PPGE - UNESC 
que neste estudo em específico trata-se do Projeto Político Pedagógico (PPC) do curso de Pedagogia da Universidade Federal da Fronteira Sul (UFFS), disponível digitalmente na página do curso no site da universidade. Inicialmente foi realizada uma busca por palavras chave no documento - nesta pesquisa buscou-se as expressões "tecnologia", "informática" e "mídia" -, extraindo alguns trechos significativos do documento que relacionam estas expressões ao desenvolvimento de práticas pedagógicas articuladas ao uso de recursos tecnológicos. Ao extrair os trechos do documento, identifica-se em quais momentos a tecnologia está presente, como o perfil do egresso, grade curricular, ementa de disciplinas e infraestrutura disponibilizada aos acadêmicos do curso. A partir destes recortes textuais, analisa-se estes trechos de forma a identificar se as tecnologias mencionadas no documento enquadram-se como tecnologias enquanto recursos instrumentais a serem "dominados" pelos futuros docentes ou tecnologias enquanto possibilidades de práticas pedagógicas para o ensino dos conteúdos específicos.

\section{Referencial Teórico}

Inicialmente, é importante definir aqui alguns termos importantes abordados no decorrer do estudo, a fim de situar o leitor sobre 0 direcionamento da pesquisa realizada. Um dos conceitos chave do trabalho é o termo "tecnologia". O dicionário Houaiss traz o termo "tecnologia" como derivado do grego tékhne + lógos, e é definido como "teoria geral e/ou estudo sistemático sobre técnicas, métodos, meios e instrumentos de um ou mais ofícios ou domínios da atividade humana [...]. Técnica ou conjunto de técnicas de um domínio particular" (Houaiss, 2009, p. 1821). Lima Júnior (2003) coloca que

técnica tem a ver com arte, criação, intervenção humana e com transformação. Tecnologia, em decorrência, refere-se a esse processo produtivo, criativo e transformativo. Como já o afirmara Marx (1978), sobre o trabalho humano, o ser humano ao criar artifícios materiais e imateriais para atuar no seu meio, transformando-o, transforma, também, a si mesmo, ressignificando seu contexto e se ressignificando com ele (p. 3).

Criar Educação, Criciúma, v. 6, n²2, julho/novembro 2017.-PPGE - UNESC 
Assim, percebemos que a tecnologia engloba um imenso conjunto de técnicas, processos, ferramentas e equipamentos, nas mais diversas esferas humanas. Podemos dizer assim que até a mais isolada aldeia possui suas tecnologias de sobrevivência, por mais simples que sejam aos nossos olhos. Kenski (2007) afirma que a tecnologia é tão antiga quanto a espécie humana, sendo que a tecnologia representa um forma de poder que distingue os grupos sociais. Para a autora, existe uma relação próxima entre tecnologia, poder, conhecimento e educação, sendo campos de extrema importância para 0 desenvolvimento das sociedades desde o início dos tempos.

Outro conceito importante a ser definido é o de formação docente. Segundo o exposto na Resolução $n^{\circ}$ 2, de $1^{\circ}$ de julho de 2015, que define as Diretrizes Curriculares Nacionais para a formação inicial em nível superior (cursos de licenciatura, cursos de formação pedagógica para graduados e cursos de segunda licenciatura) e para a formação continuada, "a formação docente inicial e continuada para a educação básica constitui processo dinâmico e complexo, direcionado à melhoria permanente da qualidade social da educação e à valorização profissional [...]" (BRASIL, 2015).

Para Ferreira (2003),

muitos tem entendido a formação de professores como um processo contínuo resultante da inter-relação de teorias, modelos e princípios extraídos de investigações experimentais e regras procedentes da prática que possibilitariam 0 desenvolvimento profissional do professor. (DARSIE; CARVALHO, 1998 apud FERREIRA, 2003, p. 25)

Desta forma, podemos inferir que a formação docente abrange as diversas interações de ensino-aprendizagem que o professor desenvolve ao longo de sua carreira profissional. Para fins de continuidade futura desta pesquisa, este estudo irá abordar principalmente a formação inicial de professores, que é aquela proferida ao docente durante seu curso de licenciatura. É dado destaque à esta formação por ser a iniciação do cidadão como educador, quando seus caminhos docentes poderão ser moldados para atuação na educação básica, a base primordial da educação no país.

Criar Educação, Criciúma, v. 6, n²2, julho/novembro 2017.- PPGE - UNESC 
Unindo os dois termos centrais desta pesquisa, temos a formação de professores para uso das tecnologias educacionais. É importante ressaltar que esta formação para uso das tecnologias deve ser crítica e reflexiva, pois como ressaltado por Richit (2014), o uso das tecnologias em educação não deve ser concebido apenas como instrumento atrativo ao estudante, mas sim como uma potencialidade de "apropriação do contexto histórico e culturalmente produzido pela humanidade e de produção de novos conhecimentos" (p. 25).

A tecnologia está nos mais diversos processos de desenvolvimento humano, sendo que a fala e a escrita também são tecnologias. Neste sentido, podemos relacionar o conceito de tecnologias intelectuais ou tecnologias da inteligência, definidas por Lévy (1993) como os instrumentos culturais e políticos que constituem a inteligência dos grupos sociais em cada momento histórico. Assim, cada momento histórico desenvolveu suas técnicas e tecnologias intelectuais, que jamais é dissociada do contexto histórico e político de cada sociedade.

Para Castells e Cardoso (2005, p. 17 apud MONTEIRO, 2014, p. 491), "a tecnologia não determina a sociedade: é a sociedade. A sociedade é que dá forma à tecnologia de acordo com as necessidades, valores e interesses das pessoas que utilizam as tecnologias".

$\mathrm{Na}$ abordagem das tecnologias intelectuais, percebemos que as tecnologias surgem e se modificam sempre com o propósito de contribuir com o desenvolvimento dos grupos sociais. É importante que sejamos cidadãos ativos no processo evolutivo das tecnologias, uma vez que

\begin{abstract}
quando o ser humano aprendeu a linguagem, ele aprendeu não só como ouvir, mas também falar. Quando se alfabetizou, aprendeu não somente a ler mas também a escrever. E como estamos nos movendo em direção a uma incrível realidade digital, nós devemos aprender não só a usar os programas mas a fazê-los" (RUSHKOFF, 2010 apud TIC EDUCAÇÃO, 2015, p. 71)
\end{abstract}

As tecnologias intelectuais também possuem um papel de extrema importância no desenvolvimento da educação e ciência. São várias as possibilidades do uso das tecnologias digitais para o compartilhamento de experiências, colaboração em projetos, busca, produção e disseminação do Criar Educação, Criciúma, v. 6, ำ2, julho/novembro 2017.- PPGE - UNESC 
conhecimento. Desta forma, estando as tecnologias presentes no meio cultural social, a educação pode fazer uso dela para potencializar seu alcance, facilitar seus processos e estimular o papel de autoria de alunos e professores na produção de conhecimento.

A incorporação das tecnologias no ambiente escolar tem avançado consideravelmente nos últimos anos, mas alguns pontos ainda permanecem como desafios, principalmente no que se refere a formação de professores para uso das tecnologias nas atividades de ensino e aprendizagem. O desafio da educação, seja enquanto escola, família ou sociedade, é desenvolver habilidades para o uso crítico de todas estas possibilidades digitais.

Entrelaçado ao processo de transformação social e cultural deflagrado pelas tecnologias, consolida-se um movimento de pesquisas que dedicam-se a compreender as implicações da presença e incorporação das tecnologias nos processos educativos. Dentre esses estudos destacam-se aqueles focalizados na formação do professor para uso das tecnologias na prática pedagógica e as dimensões abarcadas por essa formação. É nesse movimento que a partir de meados de 2005, intensificam-se os estudos na área de tecnologias educacionais que se utilizam do conceito TPACK (TechnologicalPedagogicalContentKnowledge), que em uma tradução livre representa o Conhecimento Tecnológico e Pedagógico do Conteúdo, tendo como base teórica Lee Shulmann. Este conceito surge por meio dos estudos de Schulman nos anos de 1986 e 1987, e baseia-se no pressuposto de utilização das Tecnologias da Informação e da Comunicação como apoio às estratégias pedagógicas para ensinar o conteúdo curricular, o que implica em uma atuação docente que incorpore "conhecimentos e atitudes como flexibilidade e fluência da tecnologia, da pedagogia, do conteúdo curricular e do contexto envolvido sendo que cada componente influencia diretamente no outro" (CIBOTTO; OLIVEIRA, 2013, p. 1).

$\mathrm{Na}$ abordagem de Shulman (1986), apresentada aqui por meio dos estudos de Koehler e Mishra (2009), Cibotto e Oliveira (2013) e Richit (2015), são destacados três eixos principais do conhecimento: Conhecimento de Conteúdo (CK - ContentKnowledge), Conhecimento Pedagógico (PK Criar Educação, Criciúma, v. 6, №2, julho/novembro 2017.- PPGE - UNESC 

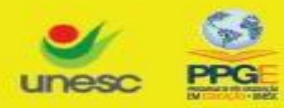

Tecnológico

(TK

TechnologicalKnowledge). O conceito de TPACK, aborda o eixo central da interação entre estes três conhecimentos.

Como é destacado por Cibotto e Oliveira (2013), o TPACK vai além das múltiplas interações entre os três elementos principais do conhecimento, abordando o ensino de conteúdo curriculares, utilizando técnicas pedagógicas, métodos ou estratégias de ensino que se utilizam das tecnologias da informação e comunicação para ensinar o conteúdo de forma diferenciada, considerando que as tecnologias contribuem na apropriação de conhecimentos por parte dos estudantes. O principal objetivo do TPACK é a articulação dos três saberes com a finalidade de proporcionar um ensino eficiente, sem ignorar a complexidade do conhecimento individual e coletivo, de forma que o contexto de articulação destes conhecimentos também é relevante para obtenção de resultados (CIBOTTO; OLIVEIRA, 2013).

Ao destacar a importância do TPACK na constituição do conhecimento profissional docente, volta-se a atenção para a articulação deste conceito na formação de professores. Ao realizar uma análise da conjuntura atual, Cibotto e Oliveira (2013) destacam que existe um déficit na formação inicial docente com relação ao conceito do TPACK, principalmente relacionado ao ensinoaprendizagem das tecnologias educacionais (CIBOTTO; OLIVEIRA, 2013)

Esta formação inicial articulada ao uso das tecnologias permite aos docentes um ambiente propício à reflexão, discussão e avaliação sobre os limites e possibilidades do uso das tecnologias no contexto pedagógico, de forma que sua utilização em sala de aula permite, também, que estes professores estimulem nos estudantes o mesmo pensamento reflexivo e crítico de utilização das tecnologias como recurso de apropriação, produção e disseminação do conhecimento (CIBOTTO; OLIVEIRA, 2013).

Segundo Almeida e Valente (2012), a integração das tecnologias no currículo escolar permite a articulação dos contextos de formação e aprendizagem com as experiências já vivenciadas, potencializando a construção permanente de práticas intencionais que constituem significados históricos, culturais e sociais. Assim, aproximando as tecnologias do contexto Criar Educação, Criciúma, v. 6, ำ2, julho/novembro 2017.- PPGE - UNESC 
escolar será possível constituir novos caminhos para sua utilização enquanto meio de desenvolvimento cultural e social.

Neste sentido, Kenski (1997) já destacava na década de 90 a importância da atuação docente para a aprendizagem crítica utilizando as tecnologias. Para a autora,

\begin{abstract}
o domínio das novas tecnologias educativas pelos professores pode Ihes garantir a segurança para, com conhecimento de causa, sobrepor-se às imposições sociopolíticas das invasões tecnológicas indiscriminadas às salas de aula. Criticamente, os professores vão poder aceitá-las ou rejeitá-las em suas práticas docentes, tirando o melhor proveito dessas ferramentas para auxiliar o ensino no momento adequado (p. 70).
\end{abstract}

Segundo os dados apontados na pesquisa TIC Educação 2014, os professores brasileiros demonstram interesse em utilizar recursos educacionais digitais, mas nem sempre existem condições de infraestrutura e capacitação para o uso dos recursos tecnológicos com propósito pedagógico. Embora a infraestrutura tecnológica esteja avançando nas escolas brasileiras, o seu uso, bem como a sua apropriação nas práticas pedagógicas, ainda representa um desafio para projetos educacionais e políticas públicas (TIC EDUCAÇÃO, 2015).

Neste sentido ressalta-se a importância da formação docente, e tal aspecto perpassa em muito momento pelas políticas públicas de formação. $O$ país ainda está evoluindo neste sentido, e um dos primeiros passos foi a aprovação da Resolução ํㅡㄹ 2, de $1^{\circ}$ de julho de 2015, que define as Diretrizes Curriculares Nacionais para a formação inicial em nível superior (cursos de licenciatura, cursos de formação pedagógica para graduados e cursos de segunda licenciatura) e para a formação continuada. Neste documento é colocado que o exercício da docência dos profissionais da educação básica é permeado por dimensões técnicas, políticas, éticas e estéticas, "envolvendo o domínio e manejo de conteúdos e metodologias, diversas linguagens, tecnologias e inovações, contribuindo para ampliar a visão e a atuação desse profissional" (BRASIL, 2015, p. 3 [grifo meu]). 


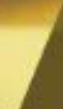

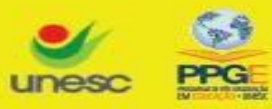

Em seu segundo capítulo, o documento trata especificamente sobre a formação dos profissionais do magistério para a educação básica, onde é colocada como uma das competências necessárias aos egressos "o uso competente das Tecnologias de Informação e Comunicação (TIC) para o aprimoramento da prática pedagógica e a ampliação da formação cultural dos (das) professores(as) e estudantes" (BRASIL, 2015, p. 6), bem como as capacidades de "desenvolvimento, execução, acompanhamento e avaliação de projetos educacionais, incluindo o uso de tecnologias educacionais e diferentes recursos e estratégias didático-pedagógicas" (Idem, p. 7) e "relacionar a linguagem dos meios de comunicação à educação, nos processos didáticopedagógicos, demonstrando domínio das tecnologias de informação e comunicação para o desenvolvimento da aprendizagem" (Idem, p. 8).

O mesmo documento também destina um tópico de seu capítulo à formação inicial docente, onde igualmente são citadas as habilidades de articular as tecnologias educacionais à prática pedagógica. Neste tópico, é reforçada a recomendação de que todos os cursos de formação inicial devem garantir

projeto formativo que assegure aos estudantes o domínio dos conteúdos específicos da área de atuação, fundamentos e metodologias, bem como das tecnologias; [...] recursos pedagógicos como biblioteca, laboratórios, videoteca, entre outros, além de recursos de tecnologias da informação e da comunicação, com qualidade e quantidade, nas instituições de formação (BRASIL, 2015, p. 9).

Sendo assim, já é previsto a inclusão de atividades formativas no âmbito das tecnologias educacionais já na formação inicial dos docentes, mas estas precisam ser muito bem planejadas e estruturadas para que possibilitem uma formação de qualidade.

O plano Nacional de Educação (PNE) 2014-2024 é um dos documentos mais recentes que traçam estratégias e políticas públicas para a difusão do uso das tecnologias educacionais, incluindo a formação de professores para tal. O PNE 2014-2014 traçou metas para o desenvolvimento da educação no período de dez anos de sua vigência. Uma das metas refere-se à alfabetização (Meta Criar Educação, Criciúma, v. 6, №2, julho/novembro 2017.- PPGE - UNESC 

como estratégias:

\begin{abstract}
selecionar, certificar e divulgar tecnologias educacionais para a alfabetização de crianças, assegurada a diversidade de métodos e propostas pedagógicas, bem como o acompanhamento dos resultados nos sistemas de ensino em que forem aplicadas, devendo ser disponibilizadas, preferencialmente, como recursos educacionais abertos [...] fomentar o desenvolvimento de tecnologias educacionais e de práticas pedagógicas inovadoras que assegurem a alfabetização e favoreçam a melhoria do fluxo escolar e a aprendizagem dos(as) alunos(as), consideradas as diversas abordagens metodológicas e sua efetividade [...] promover e estimular a formação inicial e continuada de professores(as) para a alfabetização de crianças, com - conhecimento de novas tecnologias educacionais e práticas pedagógicas inovadoras, estimulando a articulação entre programas de pós-graduação stricto sensu e ações de formação continuada de professores(as) para a alfabetização (BRASIL, 2014, p. 59).
\end{abstract}

A Meta 7 aborda questões acerca da qualidade da educação, e mais uma vez os recursos tecnológicos são vistos como parte deste processo de melhoria. A estratégia 7.15 apresenta a proposta de

\begin{abstract}
universalizar, até o quinto ano de vigência deste PNE, o acesso à rede mundial de computadores em banda larga de alta velocidade e triplicar, até o final da década, a relação computador/aluno(a) nas escolas da rede pública de educação básica, promovendo a utilização pedagógica das tecnologias da informação e da comunicação (BRASIL, 2014, p. 59).
\end{abstract}

Sobre a formação de professores, o PNE 2014-2014 estabelece quatro principais frentes de atuação: Currículo: incorporação das modernas tecnologias de informação e comunicação, em articulação com a base nacional comum da educação básica; Alfabetização: formação inicial e continuada dos docentes para a alfabetização de crianças com conhecimentos de novas tecnologias educacionais; Materiais: portal eletrônico com materiais didáticos e pedagógicos suplementares, disponibilizados gratuitamente, inclusive em formato Acessível; Cursos de formação: plataforma eletrônica para a organização da oferta e das matrículas em cursos de formação inicial e continuada (MONTEIRO, 2014).

Criar Educação, Criciúma, v. 6, n²2, julho/novembro 2017.-PPGE - UNESC 
A Meta 15 do PNE 2014-2014 aborda a formação docente, e as tecnologias se fazem presente na estratégia 15.6, que se compromete a

\begin{abstract}
promover a reforma curricular dos cursos de licenciatura e estimular a renovação pedagógica, de forma a assegurar o foco no aprendizado do(a) aluno(a), dividindo a carga horária em formação geral, formação na área do saber e didática específica e incorporando as modernas tecnologias de informação e comunicação, em articulação com a base nacional comum dos currículos da educação básica [...] (BRASIL, 2014, p. 79).
\end{abstract}

Ao analisar as perspectivas tecnológicas deste documento, Monteiro (2014) observa que há uma intenção por parte das políticas públicas para fortalecer a formação de professores com o uso das tecnologias. Sendo assim, este documento pode representar um passo importante no desenvolvimento das políticas públicas para formação docente quanto ao uso das tecnologias. Cabe agora planejar ações abrangentes para que esta formação ultrapasse os cursos rápidos e as receitas prontas de aplicações tecnológicas em sala de aula, buscando promover a apropriação crítica e reflexiva dos docentes, e consequentemente, dos estudantes, entendidos enquanto agentes sociais de transformação (MONTEIRO, 2014)

\title{
Resultados
}

Ao analisar o Projeto Pedagógico de Curso de Licenciatura em Pedagogia da UFFS, a menção à tecnologia surge já na justificativa de criação do curso, destacando a importância da formação docente e o papel da escola no contexto social do país, reforçando a firmação do Conselho Nacional de Educação da compreensão da escola como: "instituição que desenvolve uma prática educativa planejada e sistemática durante um período contínuo e extenso de tempo na vida das pessoas [...] reconhecida pela sociedade como a instituição da aprendizagem e do contato com o que a humanidade pôde produzir como conhecimento, tecnologia, cultura".

Reforçando esta ideia, o PPC do curso de Pedagogia da UFFS apresenta em seus referenciais orientadores o compromisso do curso diversos Criar Educação, Criciúma, v. 6, №2, julho/novembro 2017.- PPGE - UNESC 
aspectos de desenvolvimento da sociedade, sendo um deles a "construção de ideias para o desenvolvimento científico, tecnológico, econômico, social e cultural referenciados na dignidade da pessoa, nos valores sociais do trabalho, no pluralismo político e na solidariedade humana".

Especificamente nas atividades do curso de Pedagogia descritas no PPC, o conhecimento acerca da utilização da tecnologia surge como uma das características que constituem o perfil do egresso: "Domínio dos conhecimentos, habilidades e técnicas pedagógicas: dominar as tecnologias da aprendizagem a favor do processo pedagógico; relacionar as linguagens dos meios de comunicação à educação [...]".

Percebe-se também a preocupação do curso quanto à articulação destes conhecimentos ao incluir em sua grade curricular disciplinas voltadas às tecnologias. Constituindo o núcleo de conhecimentos comuns dos cursos desta instituição, é ofertada a disciplina de Introdução à Informática, que tem por objetivo "operar as ferramentas básicas de informática de forma a poder utilizálas interdisciplinarmente, de modo crítico, criativo e pró-ativo". Também é ofertada a disciplina de Tecnologias Digitais e Educação no núcleo de disciplinas de Domínio Específico, cujo objetivo é "conhecer as possibilidades e limites das tecnologias digitais para a construção do conhecimento e utilizá-las criteriosamente em projetos educativos".

Além destas, outras disciplinas mencionam a utilização das tecnologias no desenvolvimento das atividades, como Literatura Infanto-Juvenil, destacando na ementa "Literatura e novas tecnologias", Língua Brasileira de Sinais, destacando na ementa "Tecnologias voltadas para a surdez", e Ensino de Matemática, conteúdo e metodologia, destacando na bibliografia "Interdisciplinaridade e Novas tecnologias: formando professores".

Ao descrever a infraestrutura disponível para o curso, o PPC faz referência à dois laboratórios de informática de uso geral e duas salas para aulas de informática. Além destes, destaca-se um laboratório específico, denominado como Laboratório Articulado de Docência (LADO), que apresenta como um de seus objetivos "o uso de tecnologias da informação e da comunicação e de metodologias, estratégias e materiais de apoio inovadores". Criar Educação, Criciúma, v. 6, ํ2, julho/novembro 2017.- PPGE - UNESC 


\section{CRIAR EDUCAÇÃO}

Revista do Programa de Pós-Graduação em Educação - UNESC

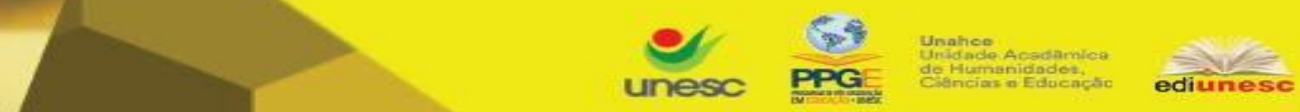

O documento ressalta que "conceber e preparar material didático (textual e visual), lançando mão dos múltiplos recursos técnicos hoje disponíveis no mercado é uma das ações previstas pelo LADO. Há que se ressaltar que o manuseio, aqui proposto, das novas tecnologias tem como finalidade última 0 exercício da reflexão a partir, de um lado, da utilização, crítica e criativa, dos conteúdos específicos de cada uma das áreas do conhecimento envolvidos nesse trabalho. E, de outro, a viabilização do diálogo entre essas diferentes áreas do conhecimento".

\section{Conclusão}

Observa-se que os primeiros dois trechos que fazem referência às tecnologias provêm de um senso geral de desenvolvimento de um campo do conhecimento humano, o conhecimento tecnológico. Porém, em ambos os casos menciona-se também o desenvolvimento cultural, sendo que na contemporaneidade as tecnologias estão imbricadas nos mais diversos aspectos culturais de nossa sociedade. Adentrando as especificidades do curso de Pedagogia, a referência à formação tecnológica articulada à formação pedagógica e de conteúdo expressa no perfil do egresso, no desenvolvimento das atividades das disciplinas e no propósito do laboratório específico, demonstra a preocupação do curso para ir além do uso instrumental da tecnologia, de forma a contribuir para uma formação inicial mais comprometida com a realidade e as necessidades sociais.

\section{Referências}

BRASIL. Plano Nacional de Educação 2014-2024: Lei ํo 13.005, de 25 de junho de 2014, que aprova o Plano Nacional de Educação (PNE) e dá outras providências. Brasília: Câmara dos Deputados, Edições Câmara, 2014. 86 p. (Série legislação; n. 125)

Criar Educação, Criciúma, v. 6, n², julho/novembro 2017.- PPGE - UNESC 


\section{CRIAR EDUCAÇÃO}

Revista do Programa de Pós-Graduação em Educação - UNESC

BRASIL. Resolução no 2, de 1ำ de julho de 2015: Define as Diretrizes Curriculares Nacionais para a formação inicial em nível superior (cursos de licenciatura, cursos de formação pedagógica para graduados e cursos de segunda licenciatura) e para a formação continuada. Ministério da Educação, 2015.

CIBOTTO, R. A. G.; Oliveira, R. M. M. A. O conhecimento tecnológico e pedagógico do conteúdo (TPACK) na formação inicial do professor de matemática. [Anais do] VIII Encontro de Produção Científica e Tecnológica: Universidade Estadual do Paraná. Campo Mourão - PR, 2013.

FERREIRA, A. C. Um olhar retrospectivo sobre a pesquisa brasileira em formação de professores de matemática. In: FIORENTINI, Dario (Org.). Formação de professores de Matemática: explorando novos caminhos com outros olhares. Campinas, SP: Mercado das Letras, 2003.

HOUAISS. Dicionário Houaiss da língua portuguesa. Rio de Janeiro: Objetiva, 2009.

KENSKI, V. M. Educação e Tecnologias: o novo ritmo da informação. Campinas, SP: Papirus, 2007.

KOEHLER, M. J.; MISHRA, P. (2009). What is technological pedagogical content knowledge? Contemporarylssues in Technology andTeacherEducation, 9(1), 60-70.

LÉVY, P. As tecnologias da inteligência: o futuro do pensamento na era da informática. Rio de Janeiro: Ed. 34, 1993.

LIMA JUNIOR, A. S. Tecnologias Intelectuais e Educação: explicitando o princípio proposicional/hipertextual como metáfora para educação e o currículo.

Criar Educação, Criciúma, v. 6, ㄲo2, julho/novembro 2017.- PPGE - UNESC 


\section{CRIAR EDUCAÇÃO}

Revista do Programa de Pós-Graduação em Educação - UNESC

Comunidades Virtuais - Grupo de Pesquisa Educação, Tecnologia e Comunicação da Universidade Estadual da Bahia.

MONTEIRO, N. A. Plano Nacional de Educação 2014-2024: as perspectivas tecnológicas nas escolas. Revista Retratos da Escola, Brasília, v. 8, n. 15, p. 489-503, jul./dez. 2014.

RICHIT, A. Formação de professores de matemática da educação superior e as tecnologias digitais: aspectos do conhecimento revelados no contexto de uma comunidade de prática online. 596f. Tese (Doutorado em Educação Matemática) - Universidade Estadual Paulista Júlio de Mesquita Filho. Rio Claro, 2015.

RICHIT, A. Percursos da formação de professores em tecnologias na educação: do acesso aos computadores à inclusão digital. In: RICHIT, Adriana (org.). Tecnologias Digitais em Educação: perspectivas teóricas e metodológicas sobre formação e prática docente. Curitiba, PR: Editora CRV, 2014.

TIC Educação 2014. São Paulo: Comitê Gestor da Internet no Brasil, 2015.

UFFS - Universidade Federal da Fronteira Sul. Projeto Pedagógico de Curso: Curso de Graduação em Pedagogia - Licenciatura. Chapecó, 2010.

VALENTE, J. F. Computadores e conhecimento: repensando a educação. Campinas: Editora (s.n.), 1993.

Apoio financeiro: Programa de Bolsas Universitárias de Santa Catarina UNIEDU / Fundo de Apoio à Manutenção e ao Desenvolvimento da Educação Superior - FUMDES.

Criar Educação, Criciúma, v. 6, №2, julho/novembro 2017.- PPGE - UNESC 